NOTAS

\title{
LEIBNIZ: EL LENGUAJE FILOSÓFICO Y EL JUEGO CON HIPÓTESIS
}

Alejandro Herrera Ibáñez*

RESUMEN: En este trabajo se examina la concepción metodológica de Leibniz en cuanto a la naturaleza del lenguaje filosófico y el planteamiento de la hipótesis relacionadas con visiones coherentes y plausibles del mundo.

PALABRAS ClAVE: Leibniz, lenguaje filosófico, hipótesis, teoría, estilo filosófico.
ABSTRACT: In this work, we will investigate Leibniz's methodology regarding the nature of philosophical language and the formulation of the hypotheses associated with coherent and plausible world views.

KEYWORDS: Leibniz, philosophical language hypothesis, theory, philosophical style.

RECEPCIÓN: 11 de abril de 2013.

ACEPTACIÓN: 25 de marzo de 2014.

* Instituto de Investigaciones Filosóficas, UNAM. 


\title{
LEIBNIZ: EL LENGUAJE FILOSÓFICO Y EL JUEGO CON HIPÓTESIS
}

\author{
Los filósofos a menudo piensan sólo lo \\ que otros hombres piensan, \\ pero poniendo atención a lo que otros \\ han pasado por alto. \\ Leibniz
}

$\mathrm{E}_{\mathrm{n}}$ ción metodológica de Leibniz en cuanto a la naturaleza del lenguaje filosófico y el planteamiento de hipótesis relacionadas con visiones coherentes y plausibles del mundo. La riqueza y la importancia del pensamiento de Leibniz, en torno a la filosofía, es enorme. Por un lado, encontramos en su obra una serie de recomendaciones metodológicas explícitas, y por otro, podemos, mediante el examen de su forma de trabajo, colegir cómo deben hacerse, según él, las teorías filosóficas. Leibniz es muy explícito sobre cómo piensa que debe ser el lenguaje filosófico. Expondré estas

1 "Prefacio a una edición de Nizolio", en Leroy E. Loemker (ed.), Leibniz, G.W. Philosophical Papers and Letters, 1976², Dordrecht, Reidel, p. 124. ideas en la primera parte de este artículo. En la segunda sección mostraré que para Leibniz una teoría filosófica debe partir de hipótesis que cumplan con ciertas normas. De cumplir con éstas, las hipótesis dejarán de serlo.

\section{I}

Pocas veces se ha reflexionado sobre el estilo filosófico, entendiendo por éste principalmente el que se despliega en el lenguaje escrito. Leibniz es uno de los pocos filósofos, hasta donde sé, que han dedicado unas líneas al tema. En un trabajo de 1670 (tenía entonces 24 años de edad) titulado "Sobre el estilo filosófico de Ni- 
zolio", 2 Leibniz enumera las que considera las características de un buen estilo filosófico. Algunas de éstas valen, desde luego, para los discursos de otras disciplinas.

Para él, las tres principales características que debe tener el lenguaje son: claridad, verdad, y elegancia. En cuanto a la claridad, se da cuando los significados de las palabras usadas son conocidos para quien lee o escucha, o -agrega prudentemente Leibniz- "al menos para la persona atenta". Aquí surge inmediatamente una dificultad. El autor no puede controlar la capacidad de comprensión de sus lectores o escuchas. Hoy día diríamos que la claridad de las palabras empleadas y su posible comprensión están en función de la audiencia a la que se dirige el discurso. Hay una gran diferencia entre dirigirse a un público general no especializado, o a un público con especialidades diferentes de la filosófica, o a un grupo de colegas en un seminario de investigación o en una revista filosófica especializada. Cada uno de los contextos ofrece al autor una serie de supuestos que debe tener en cuenta si desea ser comprendido.

Pero Leibniz parece tener en mente, sobre todo, al público general, no

${ }^{2}$ Loemker, op. cit., pp. 121-30. La exposición que sigue en esta sección se refiere a estas páginas. Era una introducción a una reedición de Anti-Barbarus, seu de veris principiis et vera ratione philosophandi contra pseudophilosophos (Parma, 1553), del humanista italiano Mario Nizolio. a los savants con quienes sostuvo una nutrida correspondencia a lo largo de su vida. Para lograr la claridad deseada, recomienda evitar los términos técnicos y si hay que usarlos porque no siempre es posible evitarlos, deben usarse con mucho cuidado. Y no siempre es posible evitarlos, porque si siempre se usaran términos populares, el lenguaje usado sería sumamente prolijo. Pero Leibniz no duda en afirmar que en los términos técnicos hay siempre cierta oscuridad. Ignora, sin embargo, que el término técnico es introducido -cuando no se abusa-en aras de la precisión, característica que pudo haber añadido a las de claridad, verdad y elegancia. Desde luego, un gran logro sería conseguir precisión sin caer en la oscuridad. Leibniz, sin embargo, es enfático en su afirmación de que no hay nada que no pueda ser explicado en términos populares y que, cuanto más populares son los términos usados, más claro será el discurso. Para elegir los términos adecuados propone adoptar como norma la búsqueda de la popularidad más compendiosa o de la compendiosidad más popular, es decir -parafraseándolo-, la búsqueda de aquellos términos populares que no sean prolijos, y de aquellos términos no prolijos que sean más populares. Por consiguiente-dice Leibniz-siempre que estén disponibles términos igualmente populares 
y compendiosos, los términos técnicos deben evitarse. Para que no quepa duda alguna sobre su posición, Leibniz subraya que ésta es una de las reglas fundamentales del estilo filosófico, y que es frecuentemente violada, especialmente por los metafísicos y los dialécticos, pero el mismo peligro existe -añade- en la moral, la política y el derecho, puesto que estas disciplinas contienen términos incorporados al uso cotidiano y abiertos a la comprensión de todos, por lo que sería "un pecado" oscurecer las cuestiones inventando términos nuevos e inconvenientes en la mayor parte. Hay otras disciplinas -admite Leibniz- en las que llega a ser necesaria la introducción de términos técnicos. Son disciplinas como las matemáticas, la física y la mecánica, en las que se dan cuestiones que no son directamente obvias para el entendimiento y que no son frecuentes en el uso común. Leibniz no incluye la filosofía entre estas disciplinas, de modo que parecería sostener que, por especializada que sea la filosofía en cualquiera de sus ramas, debe atenerse a la regla de que "todo lo que no puede explicarse en términos populares es nada y debe ser exorcizado de la filosofía como por encanto". Dice además, en un lenguaje sumamente claro, que los términos técnicos deben ser evitados como algo peor aún que una víbora o un perro (lo que habla de posibles experiencias desafortunadas de Leibniz).

El asunto es más complejo de lo que parece, pues no hay que olvidar que, en la época de Leibniz, se hacía no filosofía a secas, sino filosofía natural, en la que la física y la ontología se entremezclaban, como sucede, por ejemplo, con la noción leibniciana de fuerza, presente tanto en su metafísica como en su dinámica. ¿Cómo separar ambas nociones exponiendo una en términos populares y la otra en términos técnicos, si lo que Leibniz buscaba era mostrar que, tanto su dinámica como su metafísica, manejaban la misma noción? Lo primero que hace es sustituir, por ejemplo, la expresión aristotélica primeras entelequias -considerado por él como término técnico- por la expresión fuerzas primitivas. ${ }^{3}$ Luego, en su metafísica trata el término fuerza como término popular, caracterizándolo mediante nociones como las de exigencia y acción, y en su dinámica caracteriza el término técnicamente mediante las nociones de masa y velocidad. De hecho, por los ejemplos que usa cuando se refiere a la filosofía, se ve que tiene constantemente presentes las investigaciones de los científicos de su época. Por otro lado, en sus críticas al uso de lenguaje oscuro se refiere, en gran parte, a los escolásticos de su tiempo,

${ }^{3}$ Ibid., p. 454. 
que abusaban del latín y rehuían la utilización de las lenguas vernáculas para no confundirse ni caer en el ridículo ante personas de buen juicio y experiencia. ${ }^{4}$

La segunda característica mencionada por Leibniz es la verdad. Su introducción es más difícil de entender si no se tiene presente que las reflexiones de la filosofía natural tenían que ver mucho con la observación. Los ejemplos que pone Leibniz no tienen que ver con la filosofía directamente. Así, acude a oraciones como "Roma está situada en el Tíber" o "El número 2 es par". En la concepción contemporánea de la filosofía tiene más importancia, para el lenguaje filosófico, la consistencia -o la argumentación consistente- que la verdad. Pero para Leibniz el discurso filosófico busca la certeza y ésta no es otra cosa, que la claridad de la verdad.

La claridad no debe ser sólo semántica, es decir, no sólo debe darse en las palabras; debe también ser sintáctica, debe darse también en la construcción de las oraciones. Los oradores y los poetas son más propensos que los filósofos a caer en este tipo de oscuridad.

${ }^{4}$ Sin embargo, Leibniz no duda en decir que "los escolásticos más antiguos son con mucho superiores a ciertos de nuestros contemporáneos en agudeza, solidez, prudencia, y hasta en su más prudente huida de cuestiones inútiles", cfr. op. cit., p. 127.
La tercera característica que Leibniz menciona es la elegancia en el discurso, pero no piensa que ésta concierna de manera especial al discurso filosófico, aunque admite que puede ser de gran utilidad para captar la atención, para mover las mentes y para imprimir las cosas en la memoria con mayor profundidad. La trata, por tanto, como un elemento retórico, en el buen sentido. Leibniz acota que el discurso es elegante cuando es agradable al oído o en la lectura. Ciertamente, si a la verdad o a la consistencia, a la claridad y a la precisión, se añade la elegancia, estaremos frente a un estilo filosófico disfrutable, en el que forma y contenido se conjuntan. A mi juicio, un ejemplo contemporáneo de elegancia en el lenguaje es la obra filosófica de Bertrand Russell.

Otro consejo de Leibniz es que, a diferencia de lo que comúnmente se sostiene, para filosofar con precisión, deben usarse sólo términos concretos. Piensa que un ejemplo de ello fue Aristóteles, quien usaba sustantivos más bien que sus abstractos (p.ej. hablaba del cuál y del cuánto en lugar de hablar de cualidad y cantidad), mientras que sus seguidores se sienten más sutiles cuando usan sólo términos abstractos. Pero esta pasión por acudir a términos abstractos ha oscurecido casi totalmente la filosofía. Los concretos, en cambio 
-piensa Leibniz- son realmente cosas, en tanto que las abstracciones son sólo modos de cosas. Está en contra de la reificación de los universales, pues argumenta que las abstracciones pueden ser de diversos grados, hasta el infinito (es decir: cualidades, cualidades de cualidades, y así sucesivamente) y pueden dar lugar a contradicciones, si se tratan como cosas. Uno de sus ejemplos es el abstracto ser (entitas) del concreto ens (ente). Si el ser fuese también un ente, sería forma de sí mismo o parte de su propio concepto, lo cual implica una contradicción. En otras palabras, Leibniz vislumbra la posibilidad de paradojas cuando las abstracciones se concretizan. Recomienda, por tanto, "abstenerse de los términos abstractos casi totalmente". Esto hace pensar en la recomendación de Kripke, no ver los mundos posibles como si fuesen visibles con un telescopio. Leibniz agrega que no ha encontrado ninguna utilidad en el uso de términos abstractos para una filosofía rigurosa; se han dado, en cambio, muchos abusos, grandes y peligrosos. Es sorprendente que Leibniz no quiera usar términos abstractos; pero hay que reparar en que, cuando dice que hay que evitarlos casi totalmente, está concediendo que pueden llegar a ser necesarios; y lo que más le preocupa es la ten- dencia a cosificar las abstracciones (e.g. "el ser", "la nada", etc.).

También aconseja que los términos, ya sean populares o técnicos, no contengan figuras del lenguaje; si se quiere recurrir a ellas, deben ser pocas y aptas. Un claro ejemplo es tomado de Suárez, quien define causa como lo que fluye ser hacia algo más. Leibniz califica de "bárbara y oscura" esta definición, señalando que incluso la construcción gramatical es mala, pues el verbo intransitivo influere es convertido en verbo transitivo. La definición incurre, además, en la violación de la regla de no explicar lo oscuro mediante lo más oscuro. Que las metáforas y el lenguaje figurado no se pueden evitar en filosofía, es un hecho. Muchos filósofos inclusive hacen uso sistemático de este recurso: la metáfora ayuda indudablemente a presentar una idea abstracta. Supongo que Leibniz no está en contra del lenguaje figurado como auxiliar del lenguaje frío y descarnado; lo que le preocupa nuevamente es el posible abuso de tal tipo de lenguaje; quizás tiene en mente que el lenguaje figurado debe ser auxiliar, pero no sustituto, del lenguaje filosófico claro y preciso. Leibniz mismo se auxilió de él cuando dijo que las mónadas (¡término técnico!) no tienen ventanas (¡lenguaje figurado!).

Desde luego, la lógica anda rondando las recomendaciones leibni- 
cianas, pues para él es la herramienta con la que podemos aprender a alcanzar y confirmar la verdad, y a utilizar los recursos para la invención y el buen juicio en filosofía. A este respecto acude a un símil ilustrador: así como la retórica consta de dos partes, una que se ocupa de la combinación elegante, bella y efectiva de las palabras y otra que se ocupa de mover las emociones; así, la lógica tiene también dos partes: una verbal, que se ocupa del uso claro, distinto y apropiado de las palabras en el estilo filosófico, y otra real, que se ocupa de ser guía y control del pensamiento.

Pero su concepción del lenguaje filosófico era mucho más ambiciosa. Deseaba crear un cálculo del raciocinio o calculus ratiocinator, un lenguaje con reglas y símbolos tan precisos que toda discusión resultara prácticamente inútil, pues quedaría resuelta mediante la aplicación de las reglas de ese cálculo. En una carta de 1679 al Duque John Frederick, le comunica:

Para hacer mis demostraciones absolutamente irrefutables y tan ciertas como cualquier cosa que se pueda probar mediante el cálculo aritmético, ofreceré un ensayo sobre mi nueva escritura o característica o, si usted lo prefiere, lenguaje. Este es, sin duda alguna, uno de los más grandes proyectos a los que jamás hombre alguno se ha dedicado. Será un instrumen- to aun más útil para la mente que los telescopios y los microscopios lo son para los ojos. Cada línea de esta escritura equivaldrá a una demostración. Las únicas falacias serán errores fácilmente detectables en el cálculo. Y éste se convertirá en el gran método para descubrir verdades, establecerlas y enseñarlas irresistiblemente una vez establecidas. ${ }^{5}$

Leibniz nunca pudo materializar este sueño, y no a causa de incapacidad intelectual para ello, sino porque -creemos ahora-tal empresa es imposible de lograr, aunque contamos con herramientas formales que nos pueden brindar ayuda y ciertas aproximaciones para resolver algunos problemas. El cálculo que Leibniz anheló fue utópico; en cambio, sus reglas para el estilo filosófico son más realistas, y por ello -con todo y su modestiamás útiles para la comunicación del pensamiento filosófico.

\section{II}

Ahora bien, Leibniz es un filósofo que hace teorías, no un filósofo que sólo plantea, resuelve o disuelve problemas. No había, además, tal tipo de filósofo antes del siglo XX. Como bien sabemos, las teorías se elaboran a partir de hipótesis; algunas se convierten en verdades de la teoría,

${ }^{5}$ Ibid., p. 261. 
hasta el surgimiento de una nueva, que brinde mejores explicaciones mediante otras hipótesis. Otras hipótesis se mantienen como tales dentro de la teoría; tienen un gran grado de aceptabilidad, aunque no están plenamente confirmadas.

En el lenguaje académico de las universidades, donde se enseña filosofía, cuando el alumno va a obtener su grado mediante una disertación, se espera de él que especifique en su protocolo o proyecto qué tesis va a defender. Se espera que sepa desde un principio cuál será su postura definitiva; en las mismas universidades, en las áreas de ciencias, se pide al alumno que en su protocolo especifique cuál es su hipótesis. Pareciera que el científico es más cauto, y que no hablará de una tesis mientras su hipótesis no esté validada por los resultados de su investigación. Los hechos, en efecto, pueden desmentir la hipótesis inicial y la investigación tomará entonces un curso diferente. En filosofía, en cambio, parece no haber hechos que directamente invaliden una hipótesis. ¿Cuál sería, entonces, el proceso de validación o invalidación de una hipótesis filosófica (si es que deseamos usar el cauto lenguaje del científico)? Si el examen de los hechos no es relevante para ello, entonces la herramienta de validación en filosofía debe ser el examen de los argumentos, de su validez, de su coherencia, de su consistencia, de su corrección, de su plausibilidad. John Passmore llega a la conclusión, en Philosophical Reasoning, de que "la filosofía es especulación controlada por la argumentación". ${ }^{6}$

Leibniz, como buen filósofo natural, acude a las hipótesis tanto en la ciencia como en la filosofía. En una carta de 1669 a su mentor Jacob Thomasius, critica a un filósofo de la época, Baghemin de Stettin, porque "no hay elegancia en sus hipótesis ni consistencia en su razonamiento, sino únicamente monstruosas opiniones". ${ }^{7}$ Podemos ver cómo considera Leibniz importantísima la consistencia en la argumentación; en cuanto a las hipótesis, el criterio de la elegancia vuelve a ser recomendado. El significado de "elegancia" parece cambiar aquí, pues la elegancia de una hipótesis, si bien puede coincidir con el criterio de la elegancia en el discurso, es eso y algo más, como veremos.

El criterio de la claridad también aparece en sus consideraciones. En la misma carta a Thomasius, señala que las hipótesis más claras son las mejores; además, será un defecto de las hipótesis asumir cosas innecesarias. En esta afirmación se ve ya su

${ }^{6}$ John Passmore, Philosophical Reasoning, $1970^{2}$, London, Duckworth, p. 26.

${ }^{7}$ Loemker, op. cit., p. 93. 
simpatía por Ockham (a quien admiraba) y su principio de economía o parsimonia. Este requerimiento para las hipótesis tiene que ver, supongo, con lo que Leibniz considera que es elegante en las hipótesis. Éstas, dice, deben ser claras y no deben ser superfluas. Armado con estos criterios, en esta primera etapa de su filosofía (antes de su viaje a París), Leibniz abraza una ontología cuyos elementos básicos son: mente, espacio, materia y movimiento. Todo es reducible a ellos y explicable en sus términos. En esta época Leibniz tiene una concepción newtoniana del espacio, viéndolo como "el locus universal de todas las cosas". Posteriormente, en su madurez (1712) abandonará esta posición para abrazar una hipótesis más económica, en la que el espacio -al igual que el tiempo-es relativo y no consiste más que un "orden de coexistencias" (y el tiempo, un "orden de sucesiones").

En su etapa preparisina también admitía honestamente que no veía ni la necesidad del vacío ni la necesidad del pleno, y que cualquiera de los dos podía formar parte de una explicación satisfactoria del mundo. Posteriormente, ya en París, armado con el principio de plenitud, sostendrá que no puede haber vacío ni en el espacio ni en el tiempo. Vemos, pues, cómo Leibniz no se casa con sus hipótesis de juventud; y también que llega a admitir la plausibilidad de hipótesis alternas, aunque posteriormente descartará una de ellas.

Años después (1682-84), ${ }^{8}$ insistirá en la importancia del principio de economía para la aceptación de hipótesis; entre varias, deben escogerse aquellas que son más simples y que pueden satisfacer muchos fenómenos con facilidad. En tal situación, dichas hipótesis deben tenerse por ciertas. Se trata de un método conjetural que asume ciertas causas, sin prueba, mostrando a priori que los hechos se siguen de ellas; y a mayor número de sucesos explicados por la hipótesis, mayor es la probabilidad de ésta. Leibniz es, sin embargo, consciente de que el éxito de una hipótesis no demuestra su verdad, pero le concede una certeza "moral". Podemos, inclusive, adoptar hipótesis menos perfectas, hasta que aparezca una mejor, que explique más felizmente los mismos fenómenos o más fenómenos con igual felicidad. No hay peligro en esto -añade Leibnizsi tenemos presente la distinción entre lo cierto y lo probable.

Pero hay un sentido del concepto de hipótesis que no admite ser definido en términos del concepto de certeza: las hipótesis son conjeturales, las certezas son apodícticas, aunque puedan ser efímeras (enten-

${ }^{8}$ Ibid., p. 283. 
diendo por "efímero" inclusive largos lapsos). En una carta de 1699 a Bernoulli, Leibniz afirma que cuando puede haber certeza es mejor no usar hipótesis. ${ }^{9}$ Se refiere a la certeza matemática y a la certeza que él llama metafísica, pues, por otro lado, concede que las hipótesis pueden proporcionarnos una certeza moral. Una de sus hipótesis más atrevidas ( ¡y a la vez más contemporáneas!) fue que el momento de la muerte no puede ser definido. Algunos años antes, en un artículo de 1695 del Journal des savants, ${ }^{10}$ Leibniz se atrevió a sostener que no sólo las almas de los animales se preservan al acaecer la muerte, sino que los animales mismos (es decir, con sus cuerpos o "máquinas orgánicas") se preservan, reducidos a dimensiones imperceptibles; y no sólo eso, pues tampoco nacen, sino que se transforman. Esta rara hipótesis le parece a Leibniz la única razonable, en vista de que no tendría sentido que las almas de los animales permaneciesen, después de la "muerte", en un caos de materia confusa, y en vista de los hallazgos de los que consideraba los mejores observadores de su época (como Leeuwenhoek, el inventor del microscopio), quienes hablaban de la resucitación de las moscas y otros fenómenos biológi-

${ }^{9}$ Ibid., p. 514.

${ }^{10}$ Ibid., pp. 453-60. cos en ese entonces sorprendentes. Esta hipótesis conllevaba para Leibniz sólo certeza moral, pues en la carta a Bernoulli, aunque afirma que en un sentido metafísico no hay tal momento como el de la muerte, y que es posible que el mismo animal sea producido más de una vez, admite sin embargo - con más prudencia que en 1695-que lo contrario es también posible, pues la razón no determina esta cuestión con facilidad y, por tanto, requiere de una investigación más a fondo. Leibniz juega con hipótesis audaces, pero probables. Un mes después de dicha carta, en otra a De Volder, afirma que cuando no se está listo para defender algo con demostraciones rigurosas, la hipótesis más recomendable será la "clara y bellamente consistente internamente y con los fenómenos". ${ }^{11}$

Un ejemplo de esta consistencia puede verse en sus reflexiones sobre la relación mente-cuerpo. Leibniz piensa que las verdaderas sustancias del mundo deben ser indivisibles, pues si no fuese así, siempre podríamos encontrar nuevos elementos mediante un proceso continuo de divisiones sucesivas. En los objetos físicos, por tanto, no pueden encontrarse los elementos básicos, las sustancias fundamentales del universo. Estas sustancias deben ser forzosamente simples y, por ello mismo,

${ }^{11}$ Ibid., pp. 514-8. 
indivisibles. Sólo así pueden constituirse en los ladrillos básicos del mundo; así, postula la existencia de las mónadas. Una vez aceptada esta hipótesis, Leibniz tiene que explicar qué sucede con los cuerpos físicos; y puesto que los únicos entes del mundo reales y sustanciales, son las mónadas, la consistencia interna le pide que sostenga que los cuerpos son fenómenos resultantes de las diferentes combinaciones de mónadas. A la tesis epifenomenista más común, según la cual las almas, o las mentes, surgen de combinaciones de elementos físicos, Leibniz opone un epifenomenismo en sentido contrario: es la materia, son los cuerpos, los que surgen como fenómenos resultantes de las combinaciones monádicas; fenómenos, sin embargo, que no son meras apariencias o ilusiones, sino enraizados en la realidad de las mónadas; "fenómenos bien fundados", será la expresión leibniciana. En este caso, sin embargo, ya no se trata de una hipótesis de carácter conjetural, sino de una consecuencia necesaria de sus supuestos metafísi$\cos$. Al tener la certeza de que los sucesos materiales son fenómenos bien fundados en las combinaciones monádicas, la hipótesis adquiere un carácter de certeza más fuerte que el que daría una hipótesis con certeza moral. En una respuesta de 1716 (el año de su muerte) a Samuel Masson, Leibniz escribe:

Confieso que mi opinión, según la cual la materia no podría ser considerada como una verdadera substancia, sorprenderá a algunos espíritus que piensan superficialmente y se inclinan a creer que "la materia es la única substancia del universo": pero mi hipótesis no es por ello menos verdadera. ${ }^{12}$

Lo mismo sucede con su teoría de la armonía preestablecida, a la que llega a llamar inclusive la "hipótesis de los acuerdos". La expresión "armonía preestablecida" le parece buena, pues sigue su norma de usar términos populares comprensibles; afirma en su respuesta a Masson que "le ha parecido a la gente bastante inteligible sin que sea preciso explicar, para que se la entienda, lo que quería decir Aristoxeno cuando llamaba al alma una armonía" (Id., 629). Sin entrar en mucho detalle, Leibniz propuso la que llamó una hipótesis para explicar la unión del cuerpo y el alma, no satisfecho con las planteadas por Descartes y Malebranche. Estas tres hipótesis fueron llamadas por Leibniz: (1) la hipótesis o sistema de la influencia mutua (Descartes); (2) la hipótesis

${ }^{12}$ Ezequiel de Olaso (ed.), Leibniz, G. W. Escritos filosóficos, 1982, Buenos Aires, Charcas, p. 631 . 
o sistema del supervisor perpetuo, o de las causas ocasionales (Malebranche); y, (3) la hipótesis o sistema del acuerdo natural de dos sustancias (Leibniz). ${ }^{13}$

Leibniz defiende su hipótesis como la mejor, concluyendo que es "la más razonable" y que "da una idea maravillosa de la armonía del universo y de la perfección de las obras de Dios". ${ }^{14}$ Lo interesante es que, después de enumerar las ventajas de su hipótesis sobre las otras dos, concluye que "podemos decir que es algo más que una hipótesis, pues difícilmente es posible explicar las cosas en alguna otra manera que sea inteligible". ${ }^{15}$ Así pues, hay hipótesis que llegan a ser tan claras, consistentes y económicas, que producen una certeza que las lleva a abandonar la categoría de las hipótesis. El problema es que Leibniz no percibe que lo que parece claro para él, puede no serlo para otras personas (incluyendo a sus pares), y que la certeza puede depender a menudo de factores extralógicos.

Finalmente, para añadir un caso más a esta enumeración que no pretende de ningún modo ser exhaustiva, la doctrina que quizás ejemplifica mejor el aspecto lúdico de la consideración de hipótesis por parte de Leibniz, es la de los mundos posi-

${ }^{13}$ Ver Loemker, op. cit., p. 494.

${ }^{14}$ Ibid., p. 458.

${ }^{15}$ Ibidem. bles, no sólo porque Leibniz juega, seriamente este juego, sino porque nos muestra a Dios jugándolo. Leibniz estaría totalmente de acuerdo con el apotegma einsteiniano: Dios no juega, en efecto, a los dados con el universo; Dios juega seriamente, en cambio, mediante un cálculo que rebasa nuestras limitadas mentes, a escoger una de entre un número infinito de posibilidades y no yerra en su elección. Siendo perfecto, efectúa un cálculo perfecto para poner en existencia el más perfecto de los mundos posibles que se encuentran, como ideas o conceptos en su mente, llamada por Leibniz "la región de las ideas". Los criterios de economía y máxima productividad son aplicados por la mente divina: Dios elige como el más perfecto el mundo que "es al mismo tiempo el más simple en sus hipótesis y el más rico en fenómenos" (Discurso de Metafísica, § 6). Pero los mundos que no serán creados tienen cierta fuerza, cierta "urgencia hacia la existencia", nos dice en 1697, en Sobre el origen radical de las cosas. ${ }^{16} \mathrm{Y}$ en 1714, dos años antes de su muerte, afirma en Los principios de la naturaleza y de la gracia:

De la suprema perfección de Dios se sigue que ha elegido el mejor plan posible en la producción del universo, un plan que combina la máxima ${ }^{16} \mathrm{Ibid} .$, pp. $486-91$. 
variedad con el máximo orden; el terreno, el lugar y el tiempo arreglados de la mejor manera posible; con el máximo efecto producido por los medios más simples; con el máximo poder, el máximo conocimiento, la máxima felicidad y bondad en las cosas creadas que el universo podía permitir. ${ }^{17}$

Puede verse que el mundo actual, el mundo creado, el mundo existente, es el más perfecto de todos los mundos posibles, en virtud de tener el mayor número de fenómenos posible y de contar con el mayor poder explicativo, a partir del menor número posible de hipótesis. Máximos resultados con mínimos medios es la fórmula divina para elegir de entre esa multitud de mundos que pugnan por existir, y de los cuales sólo uno será elegido, el más apto.

Russell comenta -congruente con su tesis de los dos Leibniz, el público y el privado- que puede inferirse de la doctrina leibniciana de los mundos posibles, que:

Queda excluido el acto de la Creación [...] y la construcción de un mundo que contenga el mayor número posible de esencias coexistentes es un problema de lógica pura. De allí se concluiría que este mundo existe por definición, sin necesidad de ningún Decreto Divino: además es parte ${ }^{17}$ Ibid., p. 639. de Dios, por cuanto las esencias existen en la mente divina. ${ }^{18}$

Son muchos los que no están de acuerdo con la interpretación de Russell; sin embargo, aunque Leibniz no haya excluido explícitamente a Dios de la elección del mejor de los mundos posibles -como hombre religioso que era-, o no se haya atrevido a hacerlo, queda la duda de si la actualización del mejor de los mundos posibles puede darse por un mero procedimiento automático de autorregulación. Lo importante es que en ambos casos existe la selección de una hipótesis y la eliminación de las demás mediante criterios definidos.

En virtud de este matemático y divino cálculo, el inencontrable país de Uqbar continuará para siempre perdido en la mente divina, y que Tlön, ese planeta desconocido y descrito en su enciclopedia de cuarenta volúmenes redactados en inglés y vueltos a publicar en una de las lenguas de Tlön bajo el título de Orbis Tertius, permanecerán para siempre en la región divina de las ideas a la que se asomó Jorge Luis Borges, ${ }^{19}$ en donde un "infinito Leibniz" dirigió, convertido en un "oscuro hombre de genio", la sociedad secreta de astró-

${ }^{18}$ Bertrand Russell, Exposición crítica de la filosofía de Leibniz, 1977, Buenos Aires, Editorial Siglo Veinte, trad. de Hernán Rodríguez, p. 11.

${ }^{19}$ Jorge Luis Borges, "Tlön, Uqbar, Orbis Tertius”, en Ficciones, 1956 (reimpr. 2006), México, Joaquín Mortiz, pp. 15-44. 
nomos, biólogos, ingenieros, metafísicos, poetas, etc., en la creación de ese hipotético mundo posible no actualizado que aun pugnando por salir de la pluma borgiana, perdió la batalla contra este mundo nuestro, incomprendido y ridiculizado por Voltaire. Y por ello no debemos temer o no podemos desear con Borges que el contacto y el hábito de Tlön desintegren nuestro mundo, el menos imperfecto de todos los posibles, ni podemos abrigar la esperanza o el temor de que los entes subsistentes meinongianos del hemisferio boreal de Tlön, con sus "objetos ideales, convocados y disueltos en un momento, según las necesidades poéticas", invadan el país del Doctor Pangloss, el ilustre mentor de Cándido.
Son, sin embargo, los poetas, los hombres de genio e imaginación, quienes, sin poder salir los mundos posibles de la mente divina por una prohibición matemática, tienen la bendita posibilidad y audacia de incursionar en esas regiones ignotas que pudieron haber existido y que nunca fueron ni serán, y de traernos fragmentos y vislumbres de lo que pudo ser y no fue, como esos metafísicos de Tlön que "no buscan la verdad ni siquiera la verosimilitud: buscan el asombro".

Dice Russell que el encanto de la filosofía "consiste en la libertad especulativa, en el hecho de poder jugar con hipótesis". Así lo hizo él, así también Leibniz. 